\title{
Trial Results on Bistatic Passive Radar Using Non-Cooperative Pulse Radar as Illuminator of Opportunity
}

\author{
Piotr Samczyński, Michał Wilkowski, and Krzysztof Kulpa
}

\begin{abstract}
The paper presents the concept of passive radar exploiting the active Air Traffic Control (ATC) radar as the source of illumination, and the primary results of the measurement campaign carried out at the DSP Laboratory of the Warsaw University of Technology. The system, built using commercial off the shelf components, was able to detect and track airliners landing at Warsaw airport. To verify the system accuracy the IFF mode $S$ messages were recorded, providing ground truth of the observed planes.
\end{abstract}

Keywords-bistatic radar, non-cooperative radar, passive radar, Passive Coherent Location, PCL.

\section{INTRODUCTION}

T HE concept of the passive radar not emitting its own electromagnetic (EM) energy but exploiting emitters of opportunity was discovered almost 80 years ago, but it is only in the last decade that the rapid development of this technology can be observed [1], [2]. The low cost nature of passive radar receivers and still growing processors computational power, caused increased interest from many different research institutions (both industry and academy). The number of passive radar projects, as well as the number of countries working on this technology is growing fast [2], [3], [4], [5], [6], [7]. In general, a basic passive radar concept based on the well known bistatic radar geometry [1], [2], [8]. This simplified geometry has been shown in Fig. 1.

In bistatic operation mode a radar transmitter is located at a distance $\mathrm{L}$ from a receiver. For target detection two signal streams are needed: the direct (illumination) signal and the surveillance signal where target echo should be present. The target is detected by correlating the signals from the reference and surveillance channels. The reference channel can be obtained using several methods. In some implementations a transmitter fully cooperate with a receiver is used. This means that a receiver is synchronized with a transmitter and the radar has full knowledge of the emitted signal including waveform, time of emission, signal frequency and phase. A noncooperative configuration is more difficult, whereby passive radar can utilize any kind of emission, but it is necessary to

This work was partially supported by the Polish Ministry of Science and Higher Education under project No O N515 552539 for the years 20102012 and partially supported by the European Union within the framework of the European Social Fund through the Warsaw University of Technology Development Programme.

P. Samczynski, M. Wilkowski, and K. Kulpa are with the Institute of Electronic Systems, Warsaw University of Technology, Nowowiejska 15/19, 00665 Warsaw, Poland (e-mails: psamczynski@ieee.org; emwilk@gmail.com; kulpa@ise.pw.edu.pl).

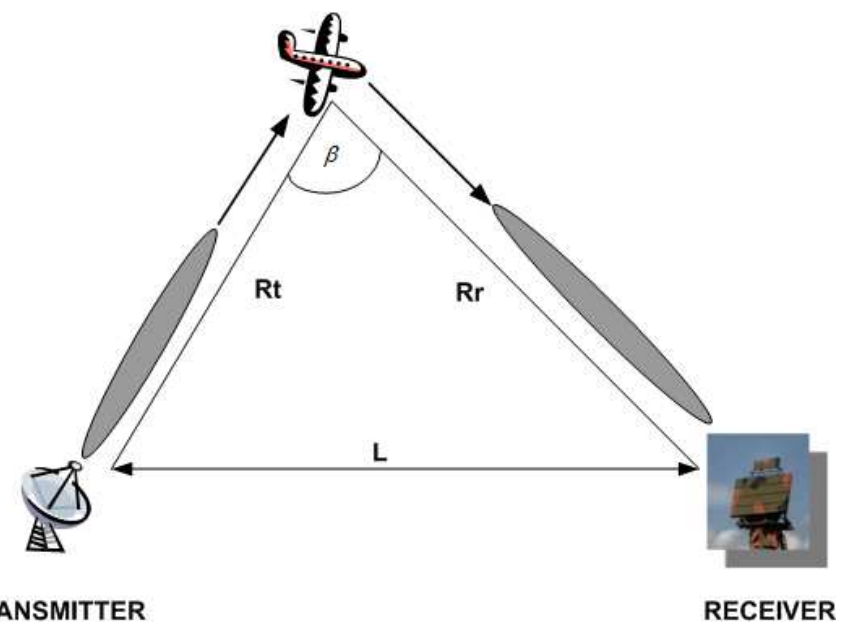

Fig. 1. Simplified bistatic radar geometry.

build at least two separate channels - one to receive the direct (transmitted) signal, and the other for surveillance - to receive the echo [1], [2]. There are a number of passive bistatic radars which use FM radio, analogue TV, DVB-T, etc. [3], [4], [5] as transmitters of opportunity.

Thanks to the lack of emission, passive radar is undetectable, contrary to classical active radar. An additional advantage of the bi- or multistatic configuration of passive radar is in the increase of probability of the detection of low RCS (Radar Cross Section) targets, including stealth targets [2], [9], [8]. The advantages listed above make the passive system very interesting for different customers (both civilian and military).

An interesting alternative to the commercial illuminators of opportunity are active radars which send high power pulses. This idea was first used in the German passive radar system Klein Heidelberg [10]. The research on that topic has been conducted worldwide [2]. The main goal of this paper is to show the current state of the research on passive radar exploiting an active air traffic control (ATC) radar as an illuminator at the Digital Signal Processing (DSP) laboratory of the Warsaw University of Technology (WUT).

\section{Bistatic RADAR With THE MECHANiCALly SCANNING TRANSMITTER ANTENNA}

One of the possible applications of the bistatic radar is to use it as a transmitter of opportunity the available pulse radars illuminators equipped with the mechannically scanning antennas. 


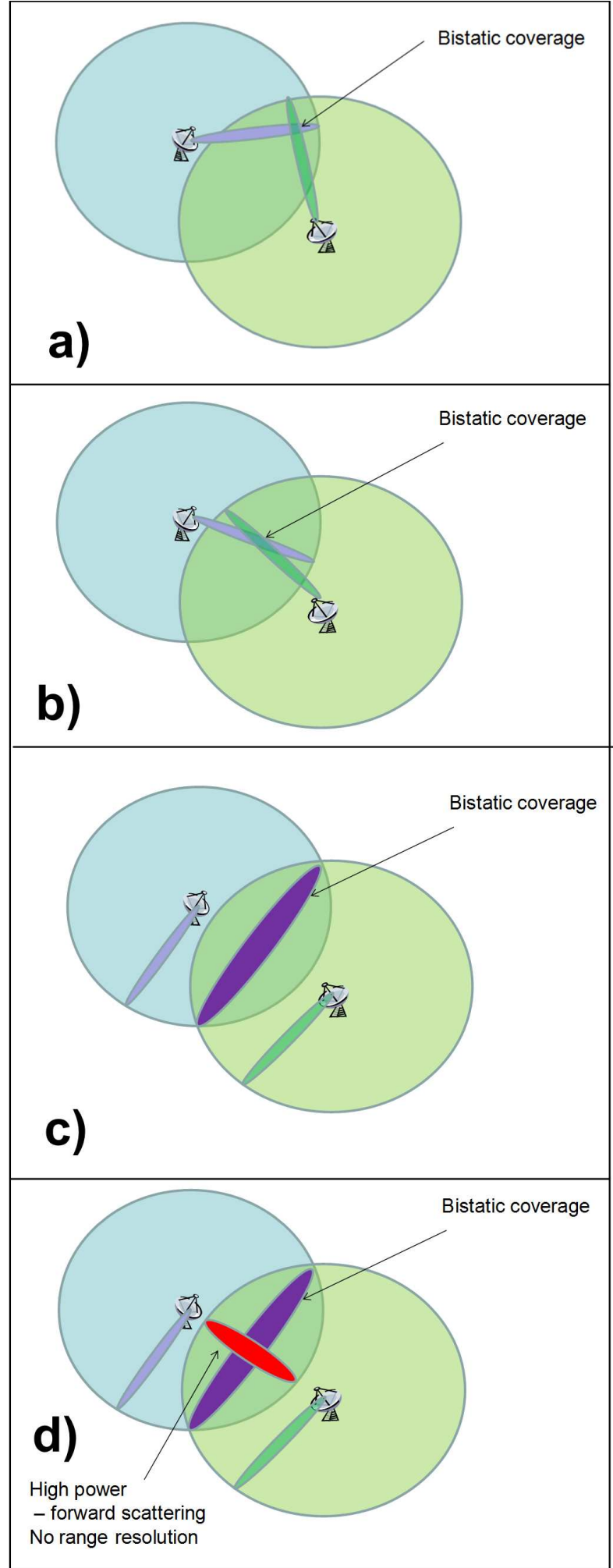

Fig. 2. The simplified geometry for the bistatic pulse radars using mechanically scanning transmitting and receiving antennas: a) bistatic coverage for $t=t_{1}$, b) bistatic coverage for $t=t_{2}$, c) total bistatic coverage, d) total bistatic coverage with marked area of no range resolution.

In such case the receiver with the fix antenna pointing in one direction or the receiver with the azimuth scanning antenna can be used. Depending on the receiver configuration different coverage areas can be achieved. The simplified geometry for the bistatic pulse radars using mechanically scanning trans- mitting and receiving antennas with marked bistatic coverage areas is presented in Fig. 2.

The simpified illustration of the bistatic coverages for the bistatic radars (see Fig. 2) shows weak capabilities of such geometry. However, such bistatic configuration has one advantage - exploited nowadays mechanically scanned radars can be used as the receivers. In such radars can be easily added the second passive mode, which would be activated by the radar operator in the special cases conditioned by the actual air traffic situation. Moreover the coverage area can be easy tuned to the diffrent areas of interest (limited to the power budget given by the range equation - see formula (1)), where based on the bistatic configuration the probability of target detection can be increased. In such configuration the maximum detection range for the passive receiver based on the beeing in service Air Trafic Control (ATC) or Airborne Early Warning (AEW) radars can be extended in comparison to the same radar working in the monostatic configuration. The simplified idea of the range extension has been shown in Fig. 3.

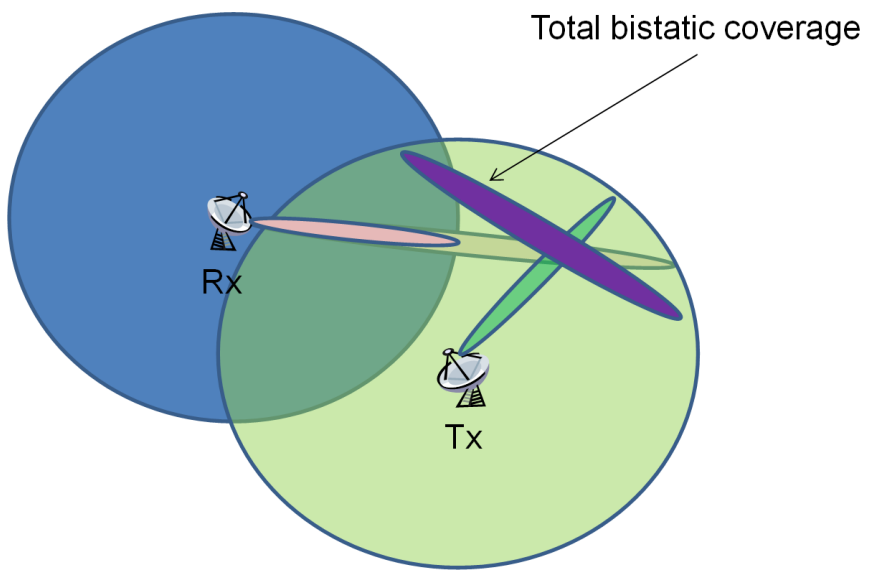

Fig. 3. The simplified geometry for the bistatic pulse radars using mechanically scanning transmitting and receiving antennas: illustration of the range extansion for the bistatic radar in comparison to the monostatic case.

Much more effective sollution to the presented bistatic configuration with receiver equiped with azimuth scanning antenna would be multichannel receiver using several directional antennas. Such a solution allows to increase the coverage area. Moreover, simple low cost antennas available on the commercial market can be used for this purposes. The simplest case of this solution is to use the receiver equipped with a single sector antenna pointing in one direction. Such a solution has been verified by the authors using the real recorded signals. The results of the trial tests are presented in the next sections of this paper.

\section{MeAsurement CAMPAign}

A measurement campaign has been carried out in the Radar and Digital Signal Processing Students' (RDSP) Laboratory on the Warsaw University of Technology (WUT) in Poland. As a passive receiver two channels synchronous Vector Signal Analyzer (VSA) has been used to record a reference signal from a radar transmitter and a signal reflected from an air 
target. To verify a detection a SBS-1 receiver has been used. The SBS-1 device decoding MODE-S signal from an aircraft transponder. As an illuminator of opportunity a non cooperative ATC radar (ASR-10SS) located on the Warsaw Airport has been used. The main parameters of ASR-10SS radar are as follows:

- carrier frequency $\left(f_{c}\right)[\mathrm{MHz}]: 2800,2801,2830,2831$;

- pulse repetition frequency $\left(f_{P R F}\right)[\mathrm{Hz}]$ : c.a. 825 ;

- pulse duration $[\mu s]: 1,100$;

- transmitted power $\left(P_{t}\right)[\mathrm{kW}]: 19.5$

- antenna gain $\left(G_{t}\right)$ [dB]: c.a. 34

The scenario and a simplified bistatic geometry is shown in Fig. 4

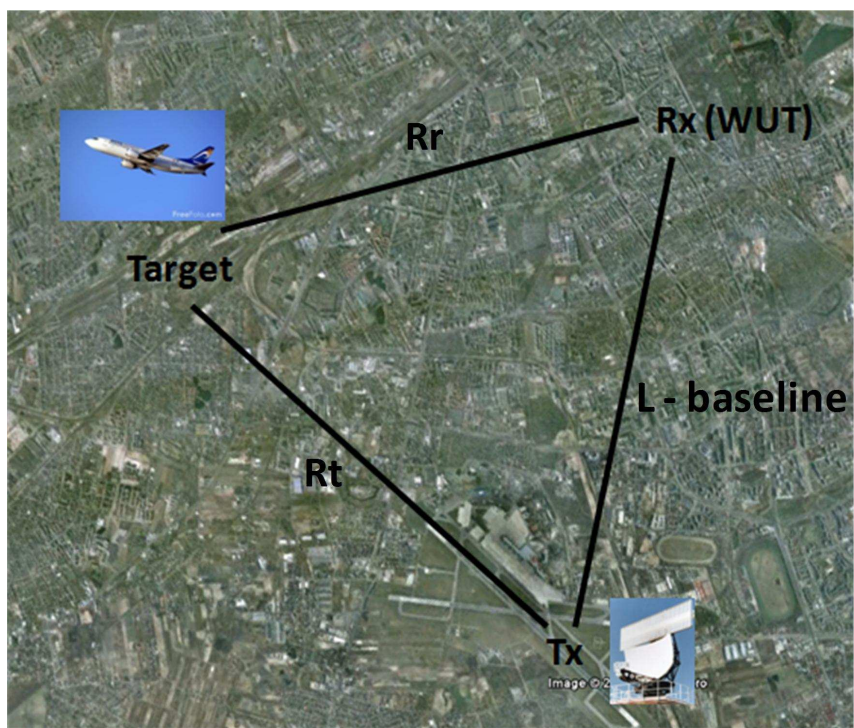

Fig. 4. Simplified bistatic geometry of WUT trials on non-cooperative bistatic radar.

In the experiment two directional antennas have been used. One antenna has been looking directly at a transmitter (Tx), which has been located in distance ca. $7 \mathrm{~km}$ from a receiver $(\mathrm{Rx})$. The second antenna has been looking at the starting aircrafts path. In Fig. 5 a map with possible to detect RCS values of observed targets has been presented.

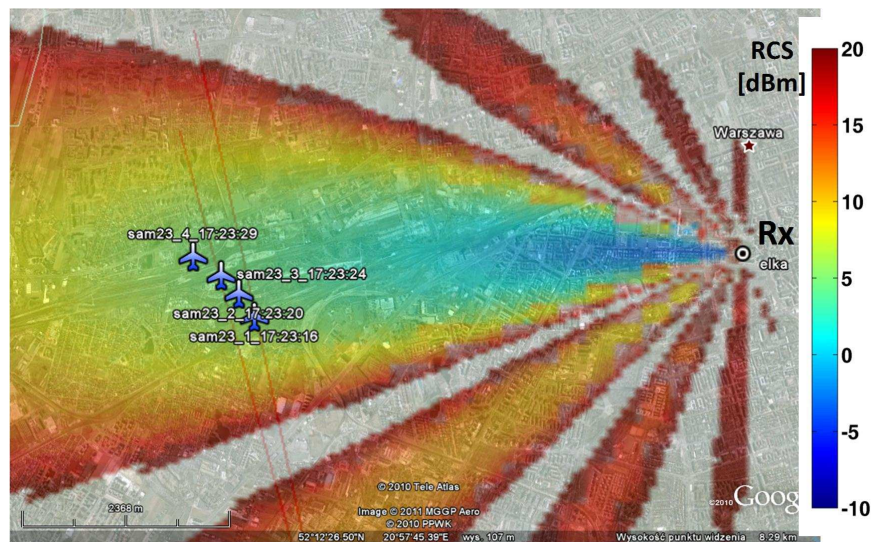

Fig. 5. Map of minimum detectable RCS for simplified bistatic geometry of WUT trials on non-cooperative bistatic radar.
The RCS map has been calculated from the range equation as follows [1], [11]:

$$
R C S\left(R_{t}, R_{r}\right)=\frac{(4 \pi)^{3} \cdot k T B \cdot L \cdot S N R}{P_{t} \cdot G_{t} \cdot G_{r} \cdot \lambda^{2}} \cdot\left(R_{t}^{2} \cdot R_{r}^{2}\right),
$$

where $R_{t}, R_{r}$ is a transmitter to target range, and receiver to target range, respectively (see Fig. 1 ), $\lambda=c / f_{c}$ is a transmitted wavelength, $k=1.38 \cdot 10^{-} 23[\mathrm{~J} / \mathrm{K}]$ is a Boltzmann's constant, $T$ is a receiving system noise temperature, $B$ is a noise bandwidth of the receiver, $L$ are a total losses in a bistatic radar system (transmitting + receiving system losses), $S N R$ is a signal to noise ratio required for target detection. Additionally in Fig. 5 a plane position received by SBS-1 device from an aircraft transponder has been indicated.

The all data registered by two channels signal analyzer has been processed further by PC. The simplified block diagram of the whole non-cooperative radar receiver is shown in Fig. 6.

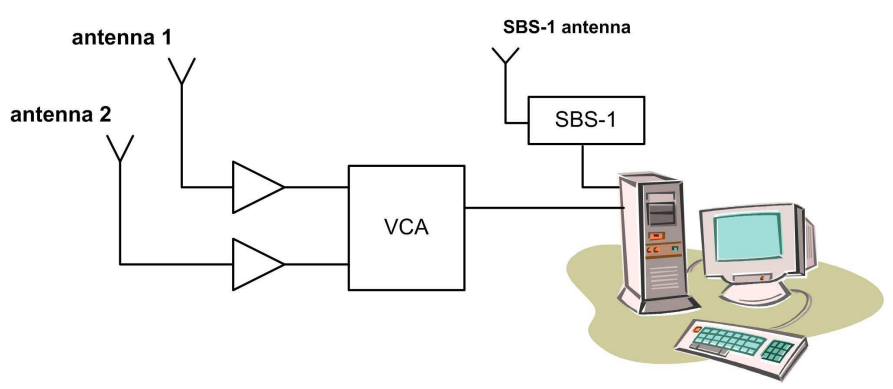

Fig. 6. Simplified block diagram of receiver system used for WUT trials on non-cooperative bistatic radar.

The recording time was ca. $20 \mathrm{~s}$, so more than one ATC radar revolution was observed. Based on the analysis of the direct signal echo from the reference antenna, the ATC antenna rotation speed was calculated. Knowing the direction of radar rotation and assuming the constant rotation angular velocity, the ATC antenna time history (angle of illumination) was reconstructed. The recorded signal from the reference antenna is presented in Fig. 7.

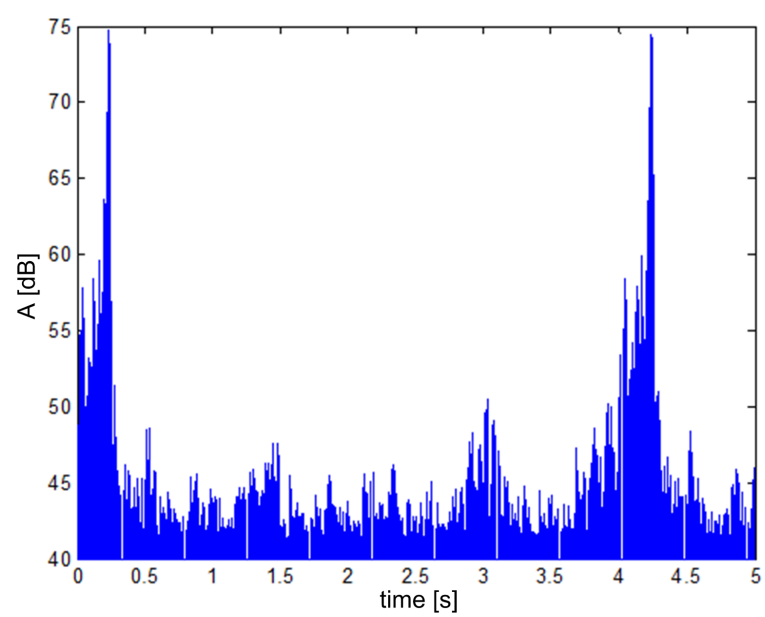

Fig. 7. Signal recorded by reference antenna. 
The detail of the investigation of the recorded signal shows that the transmitted signal burst consists of the four pulses transmitted on the single frequency, and then a different carrier frequency is selected. During registration the radar utilized four working frequencies sequentially, as depicted in Fig. 8.
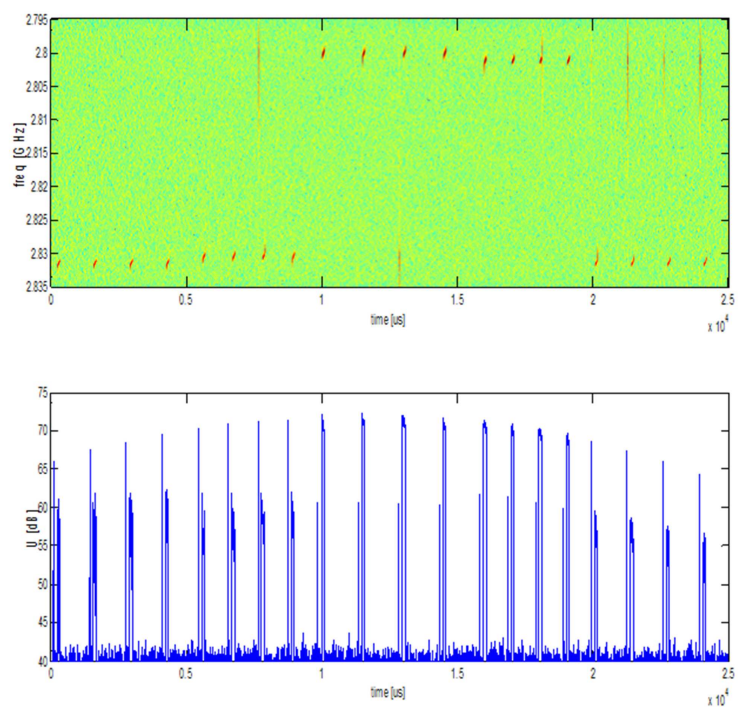

Fig. 8. Transmitted pulse bursts: time frequency plot (upper picture) and time plot (bottom picture).

Each pulse in the burst consists of the short uncoded pulse with a duration of $1 \mu \mathrm{s}$, used for short distance target detection, and chirp coded pulses of a duration of $100 \mu \mathrm{s}$, for detecting remote targets (Fig. 8). Analyzing the recorded data, both PRF and the internal signal structure have been estimated. The internal modulation of the pulses are presented in Fig. 9. For further processing only the long, chirp coded signals were used. For each frequency phase polynomial was derived from the measured data, and the set of compression filters was designed. After performing synchronization on the surveillance channel data and dividing the whole signal streams into pulse intervals, the received signal was filtered by an appropriate compression filter. This produces the rectangular range-azimuth signal matrix. The dominant elements in the surveillance signal matrix are ground/building scatterers. To perform an air target detection, ground/building clutter has to be eliminated. In literature, a number of methods dedicated to ground clutter cancelation can be found [1], [2], [11], [12] In the experiment on non-cooperative bistatic radar presented, one of the simplest solutions for clutter removal has been chosen. The pulses from a surveillance channel have been phase-corrected after match filtering. The signals processed in a surveillance channel have been multiplied by a phase estimated from a reference antenna. In the next step, four pulses in each burst have been averaged and substituted from phase-corrected pulses. This operation results in removing an echo of stationary targets from a processed range-azimuth signal matrix. The result of a range-azimuth matrix creation has been shown in section III of this paper. After clutter cancelation, CFAR algorithms can be employed for target
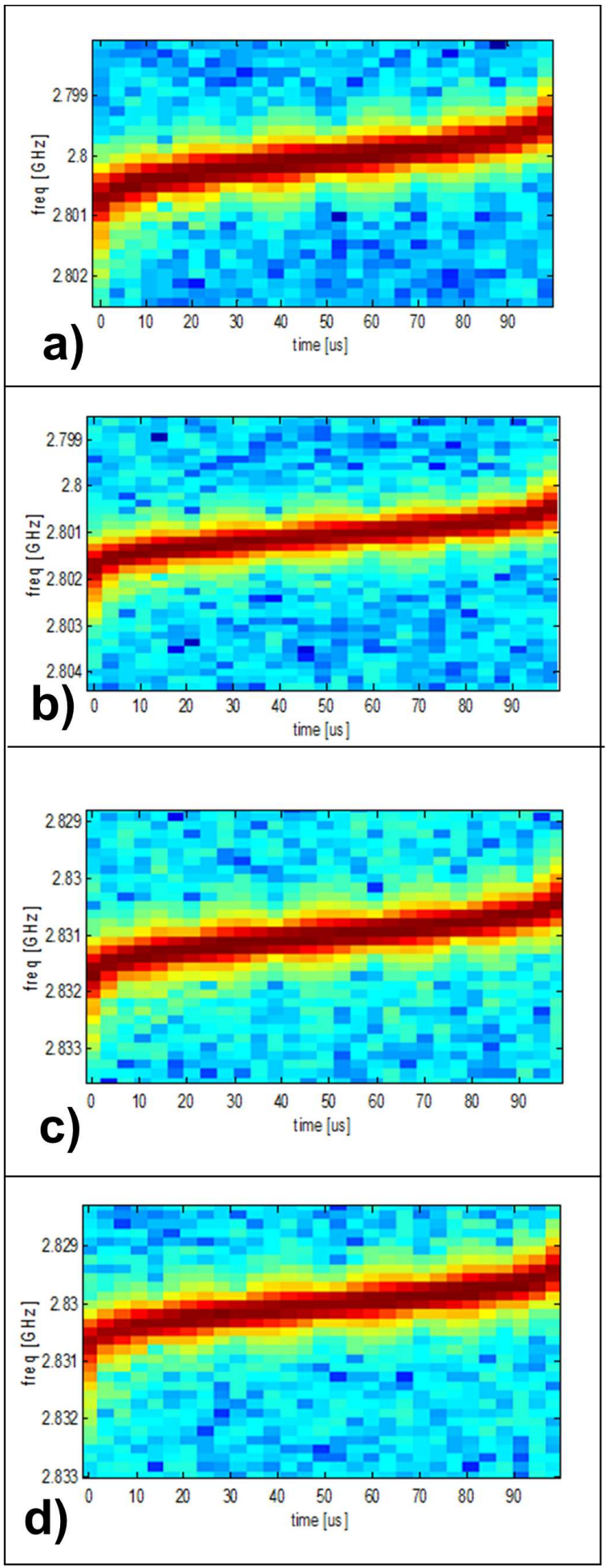

Fig. 9. The internal structure of the transmitted pulses at four different carrie frequencies $\left(f_{c}\right)$ - time-frequency plots, color coded in $\mathrm{dB}$ : a) $f_{c}=2.8 \mathrm{GHz}$, b) $f_{c}=2.801 G H z$, c) $f_{c}=2.831 G H z$, d) $f_{c}=2.830 G H z$.

detection and then tracking algorithms can be applied for tracking air targets in $R, \Theta$, or in $x, y, z$ coordinates.

\section{REsults}

The results of data processing for non-cooperative bistatic radar have been presented in Fig. 10 and Fig. 11. Fig. 10a 
a)
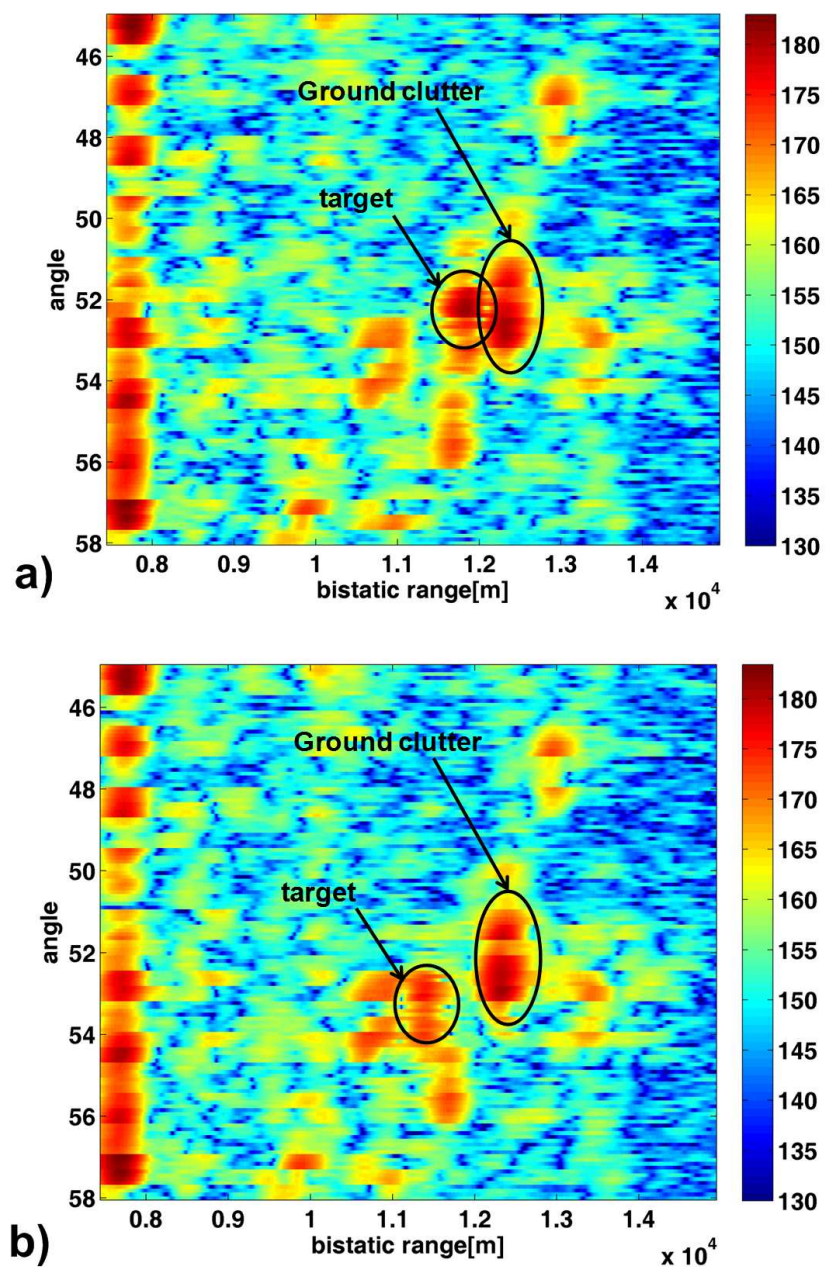

Fig. 10. Results of matched filtering for registered data for: a) first $\mathrm{Tx}$ antenna beam scan, b) second TX antenna beam scan.

and Fig. 10b present results obtained after match filtering for the different time intervals (consecutive transmitter antenna scans). The moving target is easy to recognize, however the strong reflection from the ground clutter appears. Fig. 11a and Fig. 11b show the results after ground clutter removal. An air target is clearly visible in the final result presented in Fig. 11. The airliner target detection has been verified by a plane truth path verification using a SBS-1 IFF receiver decoding a MODE-S signal from an aircraft transponder. The plane positions recorded by the SBS-1 IFF device have been presented in 5. The target positions read from the SBS-1 IFF device correspond with the positions of the target presented in Fig. 11, which has been detected using the bistatic geometry shown in 4.

\section{Conclusion}

The results presented in the paper show that passive receivers which have utilized signals from non-cooperative Air Trafic Control (ATC) or Airborne Early Warning (AEW) radars can be successfully used for an air target detection. Passive radar technology utilizing different signals coming from different transmitters of opportunity has recently entered
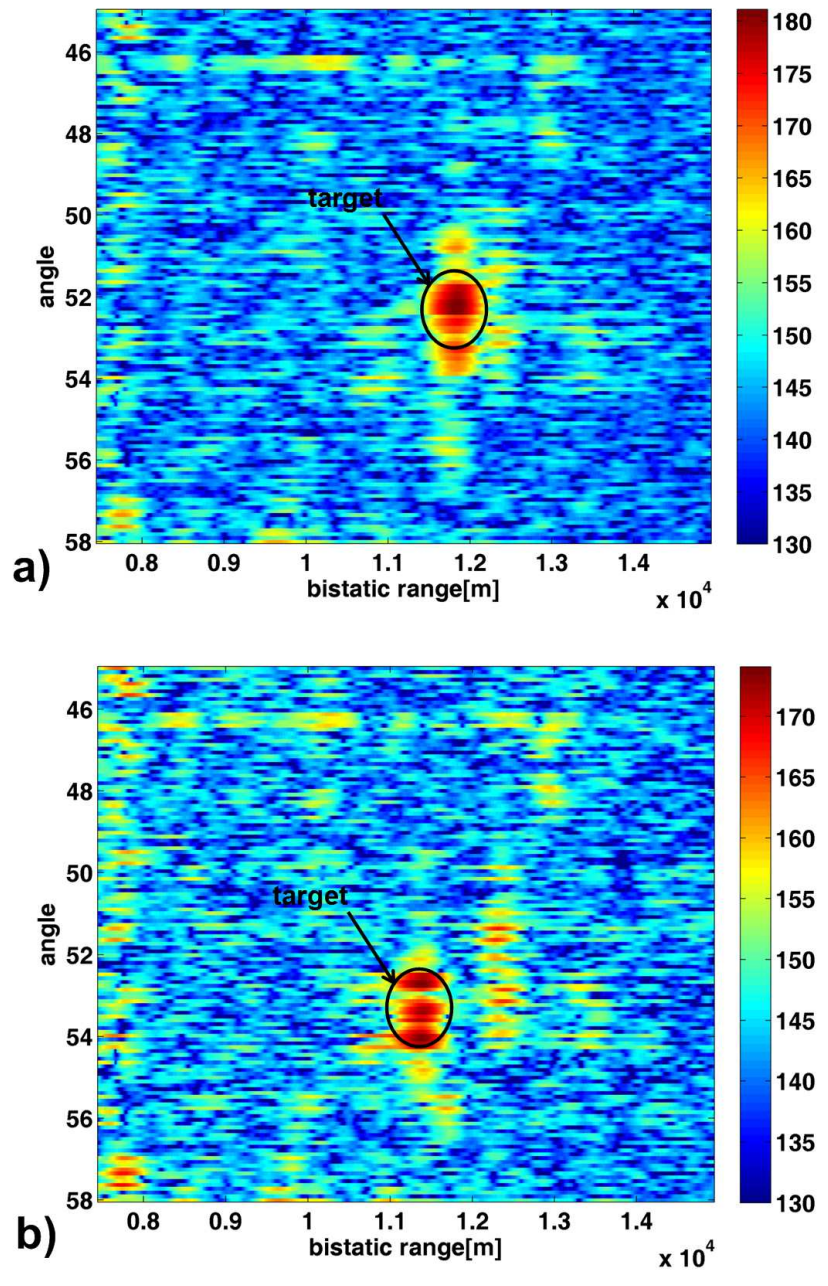

Fig. 11. Results of ground clutter removal for registered data for: a) first $\mathrm{Tx}$ antenna beam scan, b) second TX antenna beam scan.

into a stage of maturity [2]. Most passive radars utilize commercial transmitters of opportunity such as FM, DAB or DVB-T signals, which work on relatively low frequencies (radio and television signals covered ca. $80-900 \mathrm{MHz}$ of the radio frequency band). Recently, a number of multi-band passive radars using FM, DAB or DVB-T signals have been developed, which have the advantage of mutli-band operation for target detection and tracking [6], [7], [13]. The results presented show that existing passive radar systems which work with low frequencies can also be upgraded with higher frequency receivers, which will enable additional capabilities for processing signals received not only from commercial transmitters, but also from radars of opportunity such as ATC, AEW or meteorological radars.

Presented in this paper results have been obtained for the receiver using fix antenna pointing in the one direction. Such a solution provides a small bistatic coverage. In future authors plan to make an experiment with the multichannel receiver equiped with several antennas, which will extend the passive system coverage. Moreover, an interesting, however much more expensive, will be the solution with the multichannel receiver equiped with electronically scanning antenna. Such 
solution would provide extended coverage. Additionally, for such system the pulse chasing methods should be applied [14], [15], [16]. The application of the pulse chasing technique using the receiver with electronically scanning antennas and utilizing the signals coming from the non-cooperative radar as an illuminator is challenging, and possible disrupted operation is limited by growth of complexity of modern radars. While more and more radars at the modern battlefields are electronically scanned radars, the number of possible receivers will decrease in near future. More possibilities exist, when using cooperative radars as illuminators. If the radar is equipped with electronically scanned multi-beam antenna it can act either as active radar or multi-beam passive radar. It is also possible to use both modes simultaneously and construct Multiply Input Multiply Output (MIMO) system, with increase coverage and increase probability of detection.

\section{REFERENCES}

[1] N. J. Willis, Bistatic Radar, 2nd ed. SciTech Publishing Inc, 2005.

[2] K. Kulpa, J. Misiurewicz, M. Malanowski, P. Samczyński, and M. Smolarczyk, "Recent developments in passive radars," in Proceedings of Military Sensors 2009. London, UK: IQPC, 18-19 November 2009, p. CD.

[3] P. E. Howland, D. Maksimiuk, and G. Reitsma, "FM radio based bistatic radar," IEE Proc. Radar, Sonar and Navigation, vol. 152, no. 3, pp. 107115, June 2005.

[4] D. W. O'Hagan, H. Kuschel, and J. Shiller, "Passive bistatic radar analysis," vol. 7502, 2009, p. 7502Q, sPIE, Bellingham, WA, 2009.
[5] M. Malanowski, K. Kulpa, M. Mordzonek, and P. Samczyński, "PaRaDe - reconfigurable software defined passive radar," in NATO Specialist Meeting SET-136, Lisbon, Portugal, 23-25 June 2009, p. CD.

[6] M. Klein and D. Izzotte, "Operational evaluation of the HA100 during the french national day," H. Kuschel, Ed., Wachtberg, Germany, 3-4 May 2011, p. CD.

[7] A. Schröder, M. Edrich, and F. Wolschendorf, "Second-generation mobile multiband passive radar demonstrator," H. Kuschel, Ed., Wachtberg, Germany, 3-4 May 2011, p. CD

[8] H. D. Griffiths and C. J. Baker, "Passive Coherent Location radar systems. Part 1: Performance prediction," IEE Proc. Radar, Sonar and Navigation, vol. 152, no. 3, pp. 153-159, June 2005.

[9] E. Hanle, "Survey of bistatic and multistatic radar," IEE Proceedings, vol. 133, no. 7, pp. 587-595, October 1986.

[10] H. Griffiths and N. Willis, "Klein Heidelberg first modern bistatic radar system,” IEEE Trans. Aerospace and Electronic Systems, vol. 46, no. 4, pp. 1571-1588, October 2010.

[11] M. Skolnik, Radar Handbook, 2nd ed. McGraw-Hill, 1990

[12] M. I. Skolnik, Introduction to Radar Systems. McGraw-Hill, 2003.

[13] D. Poullin, M. Flecheux, and M. Klein, "3d location of opportunistic targets using DVB-SFN network : experimental results," H. Kuschel, Ed., Wachtberg, Germany, 3-4 May 2011, p. CD.

[14] D. S. Purdy, "Receiver antenna scan rate requirements needed to implement pulse chasing in a bistatic radar receiver," ieeetaes, vol. 37, no. 1, pp. 285-288, January 2001.

[15] P. Samczyński, K. Kulpa, M. Malanowski, and M. Wilkowski, "Radar bistatyczny z niekooperujcym owietlaczem - potencja i ograniczenia," in Materiay Konferencyjne IV Konferencji Naukowej Urzdzenia i Systemy Radioelektroniczne, UiSR'11, K. P. Witczak Andrzej, Ed. Rynia, Polska: WAT, 15-17 Listopad 2011, pp. 1-13, in Polish.

[16] S. Matsuda, H. Hashiguchi, and S. Fukao, "A study on multibeam pulse chasing for bistatic radar,' in Electronics and Communications in Japan, vol. 89 , no. 1 , 2006, pp. 11-21, part 1 . 